\title{
Hartman-Type and Lyapunov-Type Inequalities for a Fractional Differential Equation with Fractional Boundary Conditions
}

\author{
Imed Bachar $(\mathbb{D})$ and Hassan Eltayeb \\ King Saud University, College of Science, Mathematics Department, P.O.Box 2455, Riyadh 11451, Saudi Arabia \\ Correspondence should be addressed to Imed Bachar; abachar@ksu.edu.sa
}

Received 28 February 2020; Accepted 1 May 2020; Published 18 May 2020

Guest Editor: Thabet Abdeljawad

Copyright (c) 2020 Imed Bachar and Hassan Eltayeb. This is an open access article distributed under the Creative Commons Attribution License, which permits unrestricted use, distribution, and reproduction in any medium, provided the original work is properly cited.

We prove Hartman-type and Lyapunov-type inequalities for a class of Riemann-Liouville fractional boundary value problems with fractional boundary conditions. Some applications including a lower bound for the corresponding eigenvalue problem are obtained.

\section{Introduction}

In [1], Lyapunov established the following striking inequality:

Theorem 1. Let $q \in C([a, b], \mathbb{R})$. Assume that the problem

$$
\left\{\begin{array}{l}
\omega^{\prime \prime}+q(x) \omega=0, \quad x \in(a, b), \\
\omega(a)=\omega(b)=0,
\end{array}\right.
$$

has a solution $\omega \in C([a, b], \mathbb{R})$ such that $\omega(x) \neq 0$ for $x \in(a, b)$. Then,

$$
(b-a) \int_{a}^{b}|q(z)| \mathrm{d} z>4,
$$

and constant 4 is the best possible largest number.

It has been shown that this result serves as a good tool in the study of several properties of solutions of differential equations (such as eigenvalue problems and eigenvalue inequalities) (see, for example, [2-5] and the references therein). Many authors have worked on generalizations of classical inequalities (see, for instance, [4-16] and the references therein).

In [11], the authors use the Hahn integral operator to prove a description of new generalization of Minkowski's inequality.
In [5], the authors improve inequality in (2) by proving the following Hartman-Winter inequality:

$$
\int_{a}^{b}(b-z)(z-a) q^{+}(z) \mathrm{d} z>b-a,
$$

where $q^{+}(z)=\max (q(z), 0)$ is the nonnegative part of $q(z)$.

Inequality (3) is also known as the best Lyapunov inequality.

In [17], Ferreira considered the following fractional differential problem:

$$
\left\{\begin{array}{l}
D_{a^{+}}^{\alpha} \omega+q(x) \omega=0, \quad a<x<b, 1<\alpha \leq 2, \\
\omega(a)=\omega(b)=0,
\end{array}\right.
$$

where $q \in C([a, b], \mathbb{R})$ and $D_{a^{+}}^{\alpha}$ denotes the Riemann-Liouville fractional derivative of order $\alpha$ (see Definition 2 in the following).

The author established the following Lyapunov-type inequality for problem (4).

Theorem 2 (see [17]). Assume that problem (4) has a solution $\omega \in C([a, b], \mathbb{R})$ such that $\omega(x) \neq 0$ for $x \in(a, b)$. Then,

$$
\frac{1}{\Gamma(\alpha)} \int_{a}^{b}|q(z)| \mathrm{d} z>\left(\frac{4}{b-a}\right)^{\alpha-1} .
$$


Remark 1. Note that if we let $\alpha=2$ in (5), one obtains Lyapunov's classical inequality (2).

For the convenience of the reader, we recall the concept of fractional integral and derivative of order $\gamma \geq 0$.

Definition 1 (see $[18,19])$. The Riemann-Liouville fractional integral of order $\gamma \geq 0$ for a real-valued function $\omega$ is defined by $\left(I_{a^{+}}^{0} \omega\right)(x)=\omega(x)$ and

$$
\left(I_{a^{+}}^{\gamma} \omega\right)(x):=\frac{1}{\Gamma(\gamma)} \int_{a}^{x}(x-z)^{\gamma-1} \omega(z) \mathrm{d} z, \quad \gamma>0, x \in[a, b],
$$

where $\Gamma(\gamma)$ is the Euler gamma function.

Definition 2 (see $[18,19]$ ). The Riemann-Liouville fractional derivative of order $\gamma \geq 0$ for function $\omega$ is defined by $\left(D_{a^{+}}^{0} \omega\right)(x)=\omega(x)$ and

$$
\left(D_{a^{+}}^{\gamma} \omega\right)(x):=\left(\frac{d}{d x}\right)^{n}\left(I_{a^{+}}^{n-\gamma} \omega\right)(x), \quad \text { for } \gamma>0,
$$

where $n=[\gamma]+1$ with $[\gamma]$ the integer part of $\gamma$.

The new development in fractional calculus has attracted the attention of researchers of various disciplines. Different mathematical procedures have been considered by several authors through different research-oriented aspects of fractional differential equations (see, for instance, [20-22] and the references therein).

Our goal in this paper is to establish Hartman-type and Lyapunov-type inequalities for the following problem:

$$
\left\{\begin{array}{l}
D_{a^{+}}^{\alpha} \omega+q(x) \omega=0, x \in(a, b), \\
\omega(a)=D_{a^{+}}^{\alpha-3} \omega(a)=D_{a^{+}}^{\alpha-2} \omega(a)=\omega^{\prime \prime}(b)=0,
\end{array}\right.
$$

where $\alpha \in(3,4]$ and $q \in C([a, b], \mathbb{R})$. Some applications are given to illustrate our result.
The organization of the paper is as follows. In Section 2, we derive the explicit expression of the Green function corresponding to problem (8) and we establish some properties on it. This allows us to prove Hartman-type and Lyapunov-type inequalities for problem (8). In Section 3, we present some applications including a lower bound for the corresponding eigenvalue problem.

\section{Main Results}

2.1. Green's Function. First, we recall the following wellknown properties (see, for example, $[18,19]$ ).

Lemma 1. Let $\alpha \in(3,4)$ and $\omega \in C((a, b), \mathbb{R}) \cap L^{1}((a, b))$. Then,

(i) For $0<\gamma<\alpha, D_{a^{+}}^{\gamma}\left(I_{a^{+}}^{\alpha} \omega\right)=I_{a^{+}}^{\alpha-\gamma} \omega$ and $D_{a^{+}}^{\alpha}\left(I_{a^{+}}^{\alpha} \omega\right)=\omega$

(ii) $D_{a^{+}}^{\alpha} \omega(x)=0$ if and only if $\omega(x)=\sum_{i=1}^{4} c_{i}(x-a)^{\alpha-i}$, where $c_{i} \in \mathbb{R}$, for $i \in\{1,2,3,4\}$

(iii) Assume that $D_{a^{+}}^{\alpha} \omega \in C((a, b), \mathbb{R}) \cap L^{1}((a, b))$; then,

$$
I_{a^{+}}^{\alpha}\left(D_{a^{+}}^{\alpha} \omega\right)(x)=\omega(x)+\sum_{i=1}^{4} c_{i}(x-a)^{\alpha-i},
$$

where $c_{i} \in \mathbb{R}$, for $i \in\{1,2,3,4\}$.

Lemma 2. Let $\omega \in C([a, b])$ be a solution of problem (8). Then,

$$
\omega(x)=\int_{a}^{b} G_{\alpha}(x, y) q(y) \omega(y) \mathrm{d} y,
$$

where $G_{\alpha}(x, y)$ is Green's function of problem (8) given by

$$
G_{\alpha}(x, y)=\frac{1}{\Gamma(\alpha)} \begin{cases}\left(\frac{b-y}{b-a}\right)^{\alpha-3}(x-a)^{\alpha-1}-(x-y)^{\alpha-1}, & a \leq y \leq x \leq b, \\ \left(\frac{b-y}{b-a}\right)^{\alpha-3}(x-a)^{\alpha-1}, & a \leq x \leq y \leq b .\end{cases}
$$

Proof. Let $\omega$ be such solution. By Lemma 1, we have

$$
\omega(x)=\sum_{i=1}^{4} c_{i}(x-a)^{\alpha-i}-I_{a^{+}}^{\alpha}(q \omega)(x) .
$$

Using the fact that $\omega(a)=D_{a^{+}}^{\alpha-3} \omega(a)=$ $D_{a^{+}}^{\alpha-2} \omega(a)=\omega^{\prime \prime}(b)=0$, we obtain $c_{2}=c_{3}=c_{4}=0$ and $(b-a)^{\alpha-3} \Gamma(\alpha) c_{1}=\int_{a}^{b}(b-y)^{\alpha-3} q(y) \omega(y) \mathrm{d} y$.

Therefore,

$$
\begin{aligned}
\omega(x)= & \frac{1}{\Gamma(\alpha)} \int_{a}^{b}\left(\frac{b-y}{b-a}\right)^{\alpha-3}(x-a)^{\alpha-1} q(y) \omega(y) \mathrm{d} y \\
& -\frac{1}{\Gamma(\alpha)} \int_{a}^{x}(x-y)^{\alpha-1} q(y) \omega(y) \mathrm{d} y \\
= & \int_{a}^{b} G_{\alpha}(x, y) q(y) \omega(y) \mathrm{d} y .
\end{aligned}
$$


This ends the proof.

To get a quick perspective, in Figure 1, we have the representation of Green's function $G_{7 / 2}(x, y)$ with the contours and some projections.

One can see from Figure 1 that Green's function $G_{7 / 2}(x, y) \geq 0$ and it is nondecreasing with respect to the first variable. This important observation will be proved for $G_{\alpha}(x, y)$ with $\alpha \in(3,4]$.

Definition 3. Let $f, g:[a, b] \times[a, b] \longrightarrow \mathbb{R}$ with $f, g \geq 0$. We say that

$$
f(x, y) \approx g(x, y) \text { on }[a, b] \times[a, b]
$$

if there exists $c>0$ such that $(1 / c) g(x, y) \leq$ $f(x, y) \leq c g(x, y)$ for all $(x, y) \in[a, b] \times[a, b]$.

Remark 2. Let $\tau>0$ and $x, z \in[0,1]$. Then,

$$
\min (1, \tau)(1-z x) \leq 1-z x^{\tau} \leq \max (1, \tau)(1-z x) .
$$

Next, we establish some properties on Green's function $G_{\alpha}(x, y)$ given by $(11)$

\section{Proposition 1}

(i) On $[a, b] \times[a, b]$,

$$
G_{\alpha}(x, y) \approx(x-a)^{\alpha-2}(b-y)^{\alpha-3} \min (x-a, y-a) .
$$

(ii) On $[a, b] \times[a, b]$,

$$
\frac{\partial}{\partial x} G_{\alpha}(x, y) \approx(x-a)^{\alpha-3}(b-y)^{\alpha-3} \min (x-a, y-a) .
$$

(iii) The function $G_{\alpha}$ satisfies the following property:

$$
0 \leq G_{\alpha}(x, y) \leq G_{\alpha}(b, y), \quad(x, y) \in[a, b] \times[a, b] .
$$

Proof (i) From Lemma 2, for $x, y \in(a, b)$, we have

$$
\begin{aligned}
G_{\alpha}(x, y)= & \frac{1}{\Gamma(\alpha)}\left(\frac{b-y}{b-a}\right)^{\alpha-3}(x-a)^{\alpha-1} \\
& \cdot\left[1-\left(\frac{b-y}{b-a}\right)^{2}\left(\frac{(b-a)(x-y)^{+}}{(x-a)(b-y)}\right)^{\alpha-1}\right],
\end{aligned}
$$

where $(x-y)^{+}=\max ((x-y), 0)$.

Now, since $\left((b-a)(x-y)^{+}\right) /((x-a)(b-y)) \in[0,1]$, for $x, y \in(a, b)$, then by using Remark 2, with $\tau=\alpha-1$ and $z=\left((b-y)^{2}\right) /\left((b-a)^{2}\right) \in[0,1]$, we obtain

$$
\begin{aligned}
G_{\alpha}(x, y) \approx & (b-y)^{\alpha-3}(x-a)^{\alpha-2}[(b-a)(x-a) \\
& \left.-(b-y)(x-y)^{+}\right] .
\end{aligned}
$$

Hence, inequalities in (16) follow by observing that

$(b-a)(x-a)-(b-y)(x-y)^{+} \approx \min ((x-a),(y-a))$.

(ii) We have

$$
\frac{\partial}{\partial x} G_{\alpha}(x, y)=\frac{(\alpha-1)}{\Gamma(\alpha)} \begin{cases}\left(\frac{b-y}{b-a}\right)^{\alpha-3}(x-a)^{\alpha-2}-(x-y)^{\alpha-2}, & a \leq y \leq x \leq b, \\ \left(\frac{b-y}{b-a}\right)^{\alpha-3}(x-a)^{\alpha-2}, & a \leq x \leq y \leq b .\end{cases}
$$

Similar to case (i), by using the fact that

$$
\frac{\partial}{\partial x} G_{\alpha}(x, y)=\frac{(\alpha-1)}{\Gamma(\alpha)}\left(\frac{b-y}{b-a}\right)^{\alpha-3}(x-a)^{\alpha-2}\left[1-\left(\frac{b-y}{b-a}\right)\left(\frac{(b-a)(x-y)^{+}}{(x-a)(b-y)}\right)^{\alpha-2}\right]
$$

and applying Remark 2 with $\tau=\alpha-2$ and $z=(b-y) /(b-a) \in[0,1]$, we obtain the required result. (iii) Let $y \in[a, b]$. Since the function $x \longrightarrow(\partial / \partial x) G_{\alpha}(x, y)$ is nondecreasing on $[a, b]$, we deduce that 


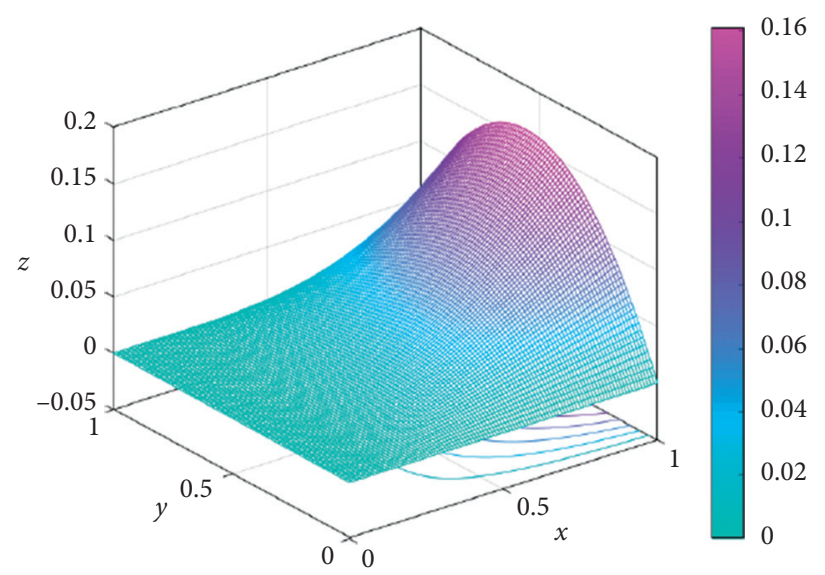

(a)

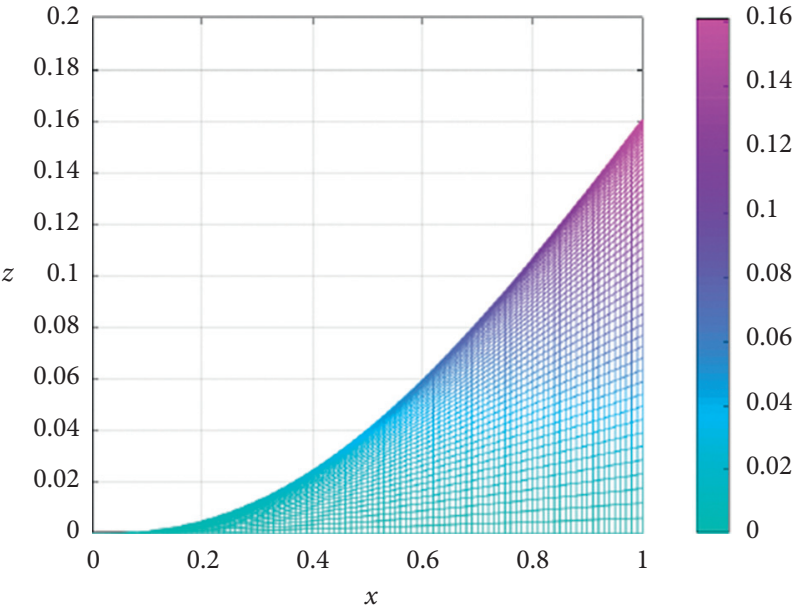

(b)

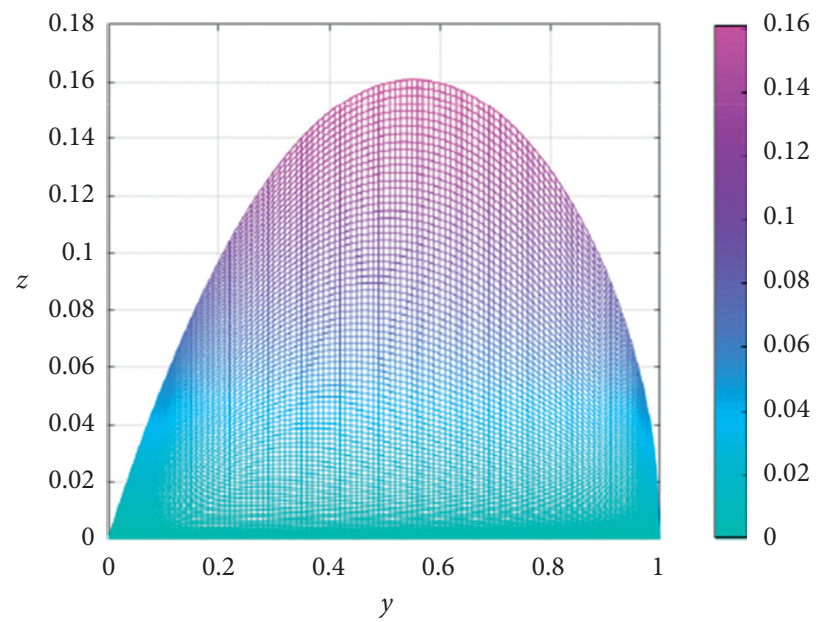

(c)

Figure 1: $G_{\alpha}(x, y)$ for $\alpha=7 / 2$. (a) $G_{\alpha}(x, y)$ and contours. (b) Projection on $x z$. (c) Projection on $y z$.

$$
0=G_{\alpha}(a, y) \leq G_{\alpha}(x, y) \leq G_{\alpha}(b, y) .
$$

This completes the proof.

\subsection{Statements and Proofs of Main Results}

Theorem 3 (Hartman-Winter-type inequality)

Let $q \in C([a, b], \mathbb{R})$. Assume that problem (8) has a solution $\omega \in C([a, b], \mathbb{R})$ such that $\omega(x) \neq 0$ for $x \in(a, b)$. Then,

$$
\int_{a}^{b}(b-z)^{\alpha-3}(z-a)(2 b-a-z) q^{+}(z) \mathrm{d} z \geq \Gamma(\alpha),
$$

where $q^{+}(z)=\max (q(z), 0)$.

Proof. From Lemma 2, we know that

$$
\omega(x)=\int_{a}^{b} G_{\alpha}(x, z) q(z) \omega(z) \mathrm{d} z, \quad x \in[a, b] .
$$

Without loss of generality, we may assume that $\omega(x)>0$ for $x \in(a, b)$.

Using (26), Proposition 1 (iii) and the fact that $q(z) \leq q^{+}(z)$, we deduce that

$$
\omega(x) \leq \int_{a}^{b} G_{\alpha}(x, z) q^{+}(z) \omega(z) \mathrm{d} z \leq \int_{a}^{b} G_{\alpha}(b, z) q^{+}(z) \omega(z) \mathrm{d} z .
$$

Hence,

$$
\|\omega\| \leq \int_{a}^{b} G_{\alpha}(b, z) q^{+}(z)\|\omega\| \mathrm{d} z,
$$

or equivalently,

$$
1 \leq \int_{a}^{b} G_{\alpha}(b, z) q^{+}(z) \mathrm{d} z .
$$

Therefore,

$$
1 \leq \frac{1}{\Gamma(\alpha)} \int_{a}^{b}(b-z)^{\alpha-3}(z-a)(2 b-a-z) q^{+}(z) \mathrm{d} z
$$

from which inequality (25) follows. 
Remark 3. Let $q \in C([a, b], \mathbb{R})$. Under the same conditions as in Theorem 3, we have

$$
\int_{a}^{b}(b-z)^{\alpha-3}(z-a)(2 b-a-z)|q(z)| \mathrm{d} z \geq \Gamma(\alpha) .
$$

By applying the previous theorem with $\alpha=4$, we obtain the following:

Corollary 1. Let $q \in C([a, b], \mathbb{R})$. Assume that the problems

$$
\left\{\begin{array}{l}
\omega^{(4)}+q(x) \omega=0, \quad x \in(a, b), \\
\omega(a)=\omega^{\prime}(a)=\omega^{\prime \prime}(a)=\omega^{\prime \prime}(b)=0,
\end{array}\right.
$$

admit a solution $\omega \in C([a, b], \mathbb{R})$ such that $\omega(x) \neq 0$ for $x \in(a, b)$. Then,

$$
\int_{a}^{b}(z-a)(b-z)(2 b-a-z) q^{+}(z) \mathrm{d} z \geq 6 .
$$

In particular,

$$
\int_{a}^{b}(z-a)(b-z) q^{+}(z) \mathrm{d} z \geq \frac{3}{(b-a)} .
$$

Corollary 2 (Lyapunov - type inequality)

Under the same conditions as in Theorem 3, we have

$$
\int_{a}^{b} q^{+}(z) \mathrm{d} z \geq \frac{\Gamma(\alpha)(\alpha-1)^{\alpha-2}}{2(b-a)^{\alpha-1}(\sqrt{(\alpha-1)(\alpha-3)})^{\alpha-3}} .
$$

Proof. By Theorem 3, we have

$$
\int_{a}^{b} f(z) q^{+}(z) \mathrm{d} z \geq \Gamma(\alpha) .
$$

where $f(z):=(b-z)^{\alpha-3}(z-a)(2 b-a-z) \geq 0$.

For $z \in(a, b)$, we have

$f^{\prime}(z)=(b-z)^{\alpha-4}\left[2(b-z)^{2}-(\alpha-3)(z-a)(2 b-a-z)\right]$.

Note that

$f^{\prime}(z)=0$ on $(a, b)$ if and only if $z=z^{*}$

$$
:=\frac{1}{\alpha-1}((\alpha-1) b-(b-a) \sqrt{(\alpha-1)(\alpha-3)}) .
$$

Furthermore, $f^{\prime}(z)>0$ on $\left(a, z^{*}\right)$ and $f^{\prime}(z)<0$ on $\left(z^{*}, b\right)$.

Hence,

$$
\sup _{z \in[a, b]} f(z)=f\left(z^{*}\right)=2 \frac{(b-a)^{\alpha-1}}{(\alpha-1)^{\alpha-2}}(\sqrt{(\alpha-1)(\alpha-3)})^{\alpha-3} .
$$
(39).

So Lyapunov-type inequality (35) follows from (36) and

Corollary 3. Let $q \in C([a, b], \mathbb{R})$. Assume that the problems

$$
\left\{\begin{array}{l}
\omega^{(4)}+q(x) \omega=0, \quad x \in(a, b), \\
\omega(a)=\omega^{\prime}(a)=\omega^{\prime \prime}(a)=\omega^{\prime \prime}(b)=0,
\end{array}\right.
$$

admit a solution $\omega \in C([a, b], \mathbb{R})$ such that $\omega(x) \neq 0$ for $x \in(a, b)$. Then,

$$
\int_{a}^{b} q^{+}(z) \mathrm{d} z \geq \frac{9 \sqrt{3}}{(b-a)^{3}} .
$$

Proof. Inequality (41) follows from (39) with $\alpha=4$.

\section{Applications}

3.1. Lower Bound for the Eigenvalues. Consider the following eigenvalue problem:

$$
\left\{\begin{array}{l}
D_{0^{+}}^{\alpha} \omega(x)+\lambda \omega(x)=0, \quad x \in(0,1), 3<\alpha \leq 4, \\
\omega(0)=D_{0^{+}}^{\alpha-3} \omega(0)=D_{0^{+}}^{\alpha-2} \omega(0)=\omega^{\prime \prime}(1)=0 .
\end{array}\right.
$$

Theorem 4. Assume that eigenvalue problem (42) has a solution $\omega \in C([a, b], \mathbb{R})$ such that $\omega(x) \neq 0$ for $x \in(a, b)$. Then,

$$
|\lambda| \geq \frac{(\alpha-2) \Gamma(\alpha+1)}{2} .
$$

Proof. By Remark 3 (with $a=0$ and $b=1$ ), we have

$$
|\lambda| \int_{0}^{1}(1-z)^{\alpha-3} z(2-z) \mathrm{d} z \geq \Gamma(\alpha),
$$

from which inequality (43) follows by observing that

$$
\int_{0}^{1}(1-z)^{\alpha-3} z(2-z) \mathrm{d} z=\frac{2}{\alpha(\alpha-2)} .
$$

3.2. Nonexistence Results. Consider the following problem:

$$
\left\{\begin{array}{l}
D_{0^{+}}^{\alpha} \omega+q(x) \omega=0, \quad x \in(0,1), 3<\alpha \leq 4, \\
\omega(0)=D_{0^{+}}^{\alpha-3} \omega(0)=D_{0^{+}}^{\alpha-2} \omega(0)=\omega^{\prime \prime}(1)=0,
\end{array}\right.
$$

where $q \in C([0,1], \mathbb{R})$. Then, we have the following result.

Theorem 5. Assume that

$$
\int_{0}^{1} q^{+}(z) \mathrm{d} z<\frac{\Gamma(\alpha)(\alpha-1)^{\alpha-2}}{2(\sqrt{(\alpha-1)(\alpha-3)})^{\alpha-3}} .
$$

Then, problem (46) has no nontrivial solution.

Proof. The assertion follows from Corollary 2.

\section{Data Availability}

No data were used to support this study.

\section{Conflicts of Interest}

The authors declare no conflicts of interest. 


\section{Acknowledgments}

The authors would like to extend their sincere appreciation to the Deanship of Scientific Research at King Saud University for funding research group no. RG-1435-043.

\section{References}

[1] A. M. Lyapunov, "Problème général de la stabilité du mouvement," in Annales de la Faculté des Sciences de Toulouse $2 e$ Série, Tome 9 (1907), p. 203-474, Vol. 17, Princeton University Press, Princeton, NJ, USA, 1947.

[2] RC. Brown and DB. Hinton, "Lyapunov inequalities and their applications," in Survey on Classical Inequalities, T. M. Rassias, Ed., pp. 1-25, Kluwer Academic Publishers, Dordrecht, London, 2000.

[3] S. Cheng, "Lyapunov inequalities for differential and difference equations," Fasciculi Mathematici, vol. 23, pp. 25-41, 1991.

[4] P. Hartman, Ordinary Differential Equations, Wiley, Hoboken, NJ, USA, 1964.

[5] P. Hartman and A. Wintner, "On an oscillation criterion of liapounoff," American Journal of Mathematics, vol. 73, no. 4, pp. 885-890, 1951.

[6] T. Abdeljawad, "A Lyapunov type inequality for fractional operators with non-singular Mittag-Leffler kernel," Journal of Inequalities and Aplications, vol. 2017, no. 1, pp. 1-13, 2017.

[7] T. Abdeljawad, "Fractional operators with exponential kernels and a Lyapunov type inequality," Advances in Difference Equations, vol. 2017, no. 1, pp. 1-13, 2017.

[8] T. Abdeljawad, J. Alzabut, and F. Jarad, "A generalized Lyapunov-type inequality in the frame of conformable derivatives," Advances in Difference Equations, vol. 2017, p. 10, 2017.

[9] T. Abdeljawad, R. P. Agarwal, J. Alzabut, F. Jarad, and A. Özbekler, "Lyapunov-type inequalities for mixed nonlinear forced differential equations within conformable derivatives," Journal of Inequalities and Applications, vol. 2018, Article ID 143, 2018.

[10] S. Dhar, Q. Kong, and M. McCabe, "Fractional boundary value problems and Lyapunov-type inequalities with fractional integral boundary conditions," Electronic Journal of Qualitative Theory of Differential Equations, vol. 43, pp. 1-16, 2016.

[11] H. Khan, C. Tunç, A. Alkhazan, B. Ameen, and A. Khan, “A generalization of Minkowski's inequality by Hahn integral operator," Journal of Taibah University for Science, vol. 12, no. 5, pp. 506-513, 2018.

[12] BG. Pachpatte, Mathematical Inequalities, Elsevier-North Holland Mathematical Library, Amsterdam, Netherlands, 2005.

[13] X. H. Tang and X. He, "Lower bounds for generalized eigenvalues of the quasilinear systems," Journal of Mathematical Analysis and Applications, vol. 385, no. 1, pp. 72-85, 2012.

[14] A. Tiryaki, "Recent developments of Lyapunov-type inequalities," Advances in Dynamical Systems and Applications, vol. 5, no. 2, pp. 231-248, 2010.

[15] A. Wintner, "On the non-existence of conjugate points," American Journal of Mathematics, vol. 73, no. 2, pp. 368-380, 1951.

[16] X. Yang, Y.-I. Kim, and K. Lo, "Lyapunov-type inequality for a class of odd-order differential equations," Journal of
Computational and Applied Mathematics, vol. 234, no. 10, pp. 2962-2968, 2010.

[17] R. Ferreira, "A Lyapunov-type inequality for a fractional boundary value problem," Fractional Calculus and Applied Analysis, vol. 16, no. 4, pp. 978-984, 2013.

[18] I. Podlubny, "Fractional differential equations," in Mathematics in Sciences and Engineering, Vol. 198, Academic Press, Cambridge, MA, USA, 1999.

[19] S. Samko, A. Kilbas, and O. Marichev, Fractional Integrals and derivative, Theory and applications, Gordon \& Breach, London, UK, 1993.

[20] R. P. Agarwal, D. O’Regan, and S. Staněk, "Positive solutions for mixed problems of singular fractional differential equations," Mathematische Nachrichten, vol. 285, no. 1, pp. 27-41, 2012.

[21] Z. Bai and T. Qiu, "Existence of positive solution for singular fractional differential equation," Applied Mathematics and Computation, vol. 215, no. 7, pp. 1-7, 2009.

[22] A. Khan, H. Khan, J. F. Gómez-Aguilar, and T. Abdeljawad, "Existence and Hyers-Ulam stability for a nonlinear singular fractional differential equations with Mittag-Leffler kernel," Chaos, Solitons \& Fractals, vol. 127, pp. 422-427, 2019. 